\title{
Brief
}

\section{Endografting facilitated by axillary-axillary bypass for distal arch aneurysm after left internal thoracic artery to left anterior descending artery bypass surgery}

Naozumi Saiki, MD, Shin Ishimaru, MD, Satoshi Kawaguchi, MD, Taro Shimazaki, MD, Yoshihiko Yokoi, MD, and Yukio Obitsu, MD, Tokyo, Japan

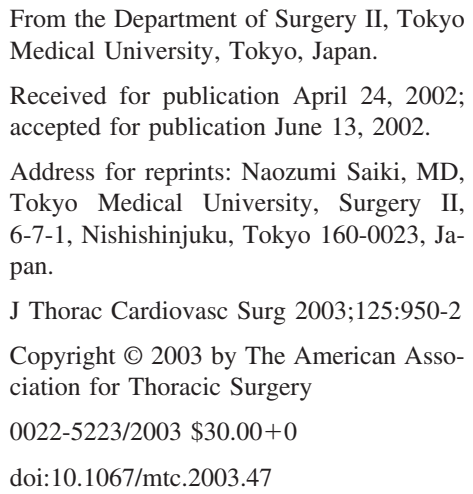

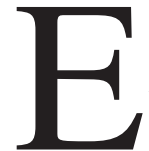
ndograft repair is a less-invasive method of treating thoracic aortic aneurysms. ${ }^{1}$ Despite advances in open surgical techniques and cardiopulmonary bypass, cerebral injury still remains a frequent postoperative complication with these procedures. We report a case of thoracic aortic aneurysm that developed after coronary artery bypass grafting of the left internal thoracic artery (LITA) to the left anterior descending artery (LAD). It was successfully managed by means of endografting and an axillary-axillary bypass procedure, so as to maintain left subclavian arterial flow. ${ }^{2-4}$

\section{Clinical Summary}

A 69-year-old man presented for stent graft treatment of a thoracic aortic aneurysm in December 2000. The patient had undergone abdominal aortic aneurysm repair 12 years earlier. He had a left hemiplegia after a stroke 4 years earlier. In June 2001, the patient had hoarseness. In June 1999, he had undergone coronary artery bypass grafting with a LITA anastomosis to the $\mathrm{LAD}$, a radial artery graft to the right posterior descending artery, and a saphenous vein graft to the obtuse marginal branch.

Computed tomography at the time of presentation showed a saccular aneurysm approximately $6 \mathrm{~cm}$ in diameter on the aortic arch (Figure 1). Because of his major cerebral infarction, an endograft repair was indicated. In this type of case, endografting normally requires occlusion of the left subclavian artery. However, we added an axillary-axillary bypass grafting to maintain the blood flow to the LITA through the left subclavian artery. Informed consent was obtained, and the procedure was performed in the operating room after achievement of general anesthesia.

The biaxillary artery and the right femoral artery were exposed. Heparin sodium (2500 units) was administered intravenously, and the activated coagulation time was maintained at greater than 200 seconds. We performed the axillary-axillary bypass procedure with a $6-\mathrm{mm}$ diameter polytetrafluoroethylene tube. Then we occluded the left subclavian artery with the occluder stent graft. The occluder stent graft was delivered through the sheath and deployed into the orifice of the left subclavian artery. A 400-cm, 0.032-inch guide wire was introduced through the right brachial artery and down to the abdominal aorta. The distal end of the guide wire was caught with a snare catheter (Amplaz goose-neck snare, Microvena Co) and picked up at the right femoral artery. A 20F sheath (Keller Timmerman Sheath, Cook Co) was introduced transfemorally over the guide wire with the tug-of-wire technique. ${ }^{5}$ The stent graft was constructed from a self-expanding Gianturco Z stent (Cook Co) and thin-wall woven polyester fabric (Ube woven-graft, Ube Industries). The stent graft diameter was $36 \mathrm{~mm}$, with a length of $170 \mathrm{~mm}$. The stent graft was delivered through the sheath and deployed into the aorta.

The postoperative course was uneventful, with no new cerebral damage or ischemic heart disease. Postoperative computed tomographic scanning showed that no aortic aneurysm was present (Figure 2). Postoperative angiography revealed that the thoracic aortic aneurysm was 

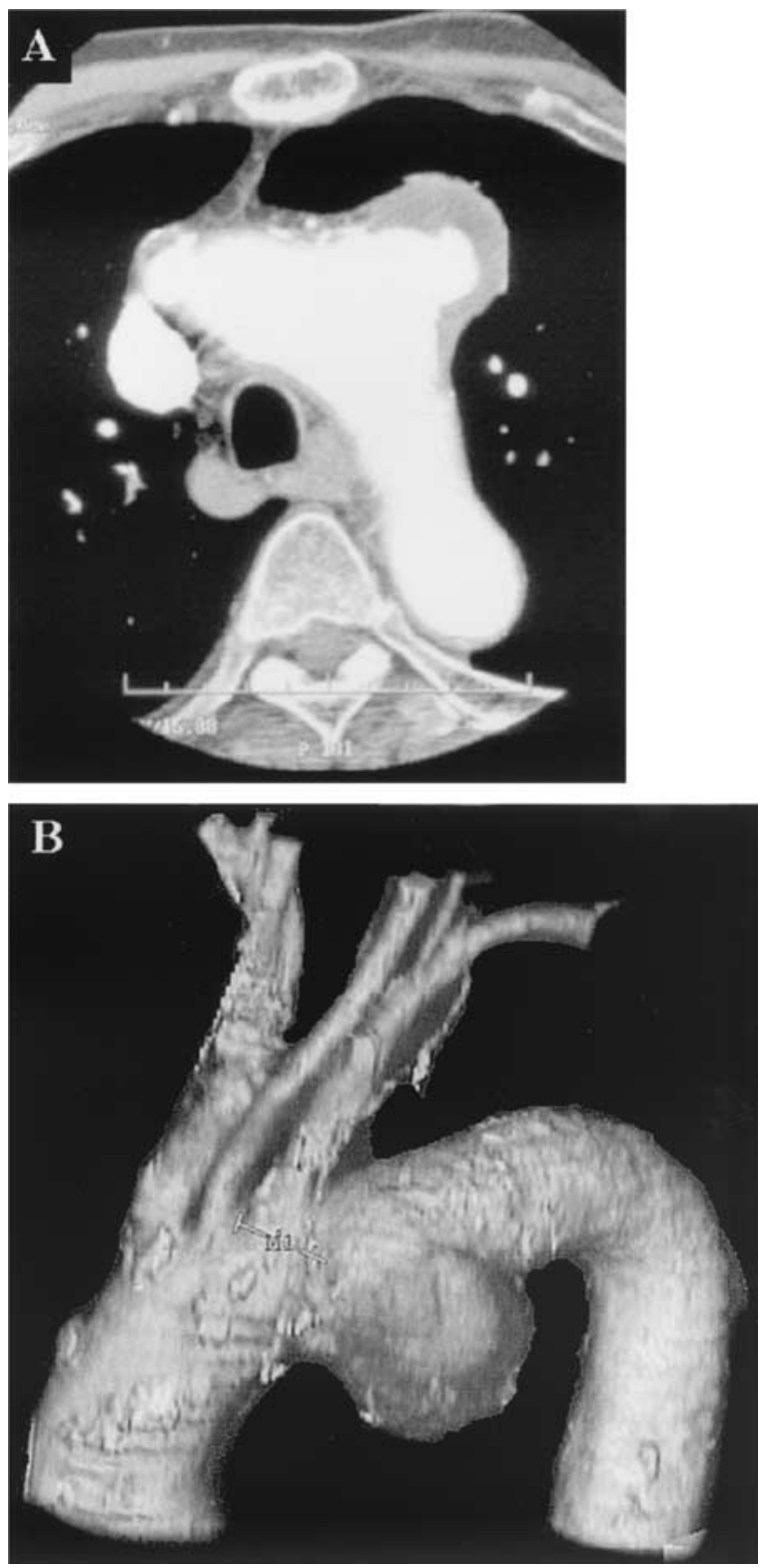

Figure 1. Preoperative computed tomogram showing the aortic aneurysm on the distal aortic arch: A, horizontal slice; B, 3dimensional computed tomogram.

completely closed by the stent graft and that the axillary-axillary bypass graft and the LITA-LAD graft were patent (Figure 3).

\section{Discussion}

An endograft repair is less invasive than conventional open surgery. In fact, conventional operation can result in life-threatening complications in patients with risk factors, such as cerebral disease and ischemic heart disease. In such patients an endograft repair should be performed. A minimum 15-mm-long proximal landing zone is required for successful endografting without an endoleak. In our case the occlusion of the left subclavian artery could

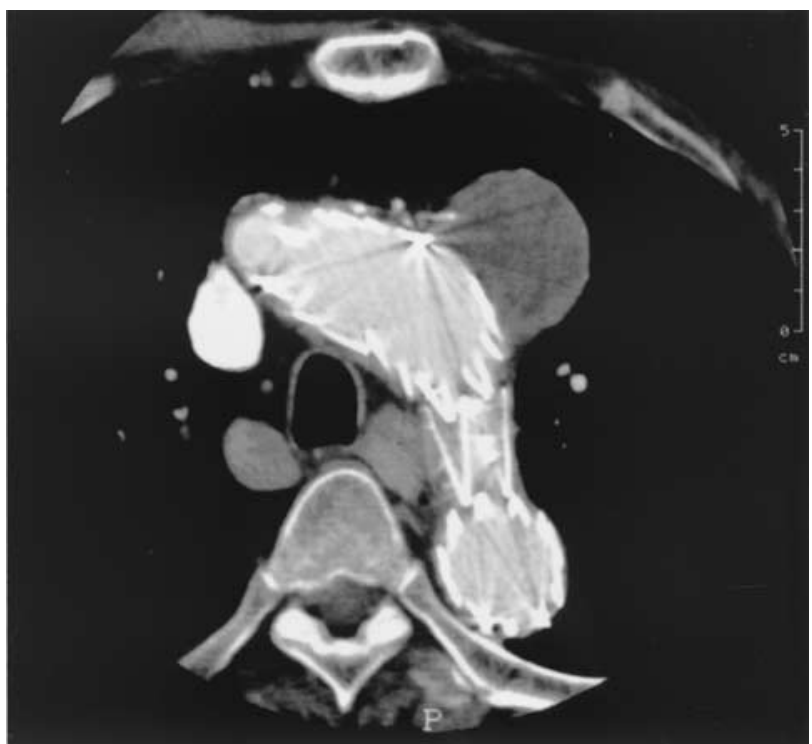

Figure 2. Postoperative computed tomogram showing thrombosis of the thoracic aortic aneurysm by means of the stent graft treatment.

produce a proximal landing zone of greater than $15 \mathrm{~mm}$, thus making endografting possible. However, we were not able to reduce the left subclavian arterial blood flow because the previous LITA-LAD bypass was patent. Therefore an axillary-axillary bypass procedure was combined with the endografting.

In the literature ${ }^{2-4}$ the axillary-axillary bypass procedure is used for subclavian and innominate artery disease because it facilitates anatomic exposure with no concern of interfering with the carotid, vertebral, and LITA circulations. The axillary-axillary bypass procedure has a reported 5-year patency rate of approximately $90 \%$. The most common complication of this procedure is transient brachial plexopathy, which occurs in $3.5 \%$ of patients; graft infection or skin erosion has been noted in $1.6 \%$ of patients. Because of collateral circulation, this extra-anatomic bypass graft does not lead to a steal phenomenon from the donor vessels. It is thought that this procedure can provide a full blood supply to the recipient artery.

Adjunctive procedures, such as the axillary-axillary bypass procedure, permit endograft repair by extending the proximal endograft landing zone. As further adjunctive procedures are designed, the indications for endograft repair will expand so as to include cases thought previously to be inoperable or very difficult to repair.

\section{References}

1. Dake MD, Kato N, Mitchell RS, et al. Endovascular stent-graft placement for the treatment of acute aortic dissection. $N$ Engl J Med. 1999;340:1546-52.

2. Mozersky DJ, Barnes RW, Sumner DS, et al. The hemodynamics of the axillary-axillary bypass. Surg Gynecol Obstet. 1972;135:925-9.

3. Lowell RC, Mills JL. Critical evaluation of axilloaxillary artery bypass for surgical management of symptomatic subclavian and innominate artery occlusive disease. Cardiovasc Surg. 1993;1:530-5. 

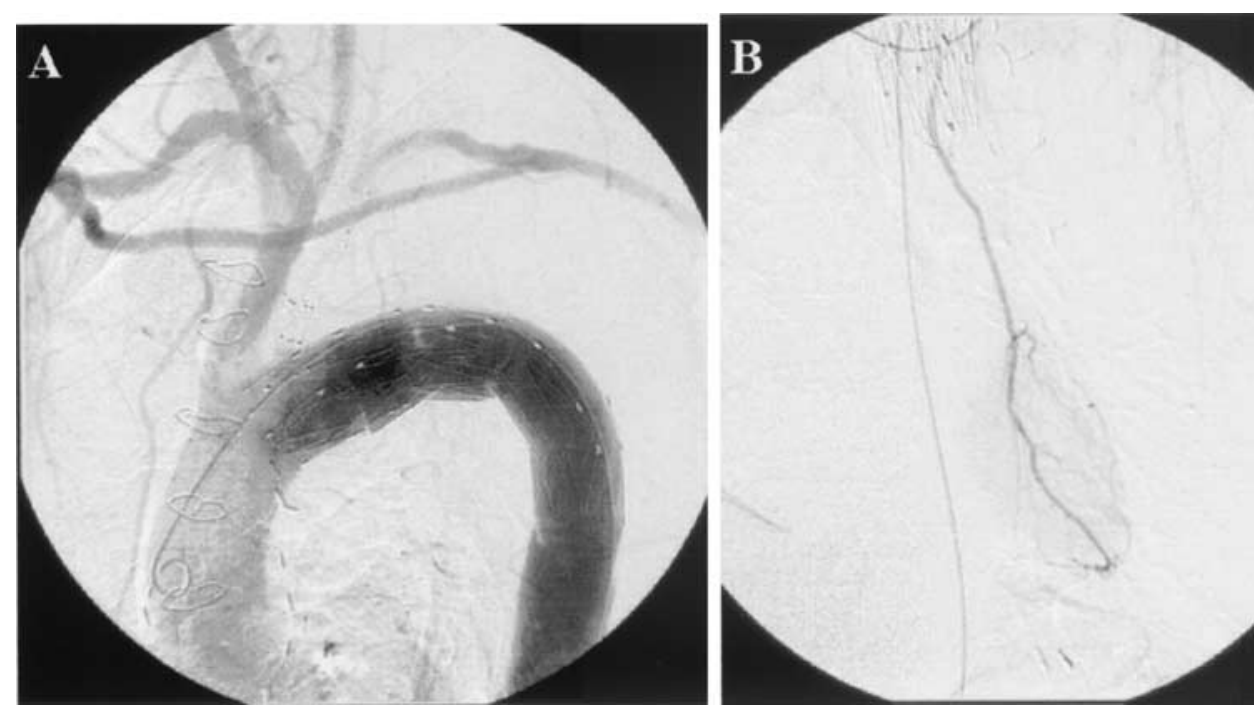

Figure 3. Postoperative angiography revealing that the thoracic aortic aneurysm was completely closed by the stent graft $(A)$ and that the axillary-axillary bypass and LITA-LAD graft were patent (A and $B)$.

4. Yamada N, Konishi Y, Matsumoto M, et al. Efficacy of axillaryaxillary bypass grafting for the subclavian artery disease. Jpn J Thorac Surg. 2000;53:1110-3.

5. Ishimaru S, Kawaguchi S, Koizumi N, Obitsu Y, Ishikawa M. Pre- liminary report on prediction of spinal cord ischemia in endovascular stent graft repair of thoracic aortic aneurysm by retrievable stent graft. J Thorac Cardiovasc Surg. 1998;115:811-8.

\section{Ascending aortic cannulation for Stanford type A acute aortic dissection: Another option}

Kenji Minatoya, MD, Matthias Karck, MD, Eugeniusz Szpakowski, MD, Wolfgang Harringer, MD, and Axel Haverich MD, Hannover, Germany

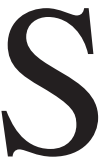

tanford type A acute aortic dissection (AADA) is still a surgical challenge. Femoral cannulation remains the standard option for surgical repairs of AADA. However, retrograde perfusion has a potential risk of embolization of atheromatous debris, extension of dissection, and malperfusion.

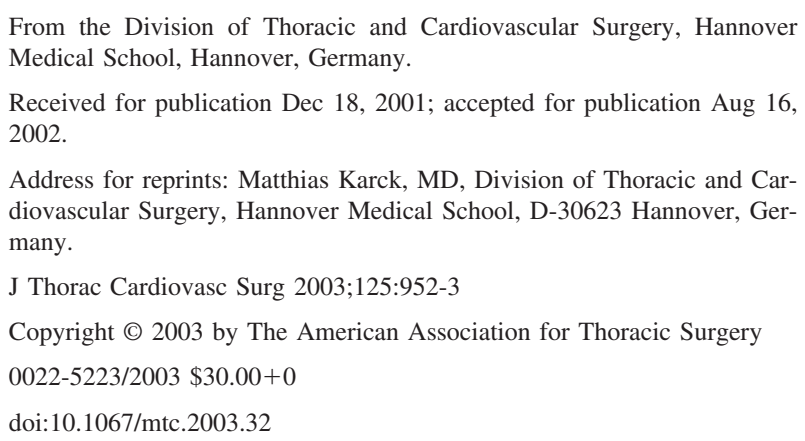

We hereby present our experiences with direct cannulation of the dissected ascending aorta in patients with AADA (Figure 1).

\section{Clinical Summary}

Between January 1998 and September 2000, 41 patients had a surgical repair of AADA with median sternotomy in our hospital. Among this population, 14 patients had ascending aortic or proximal arch cannulation to initiate cardiopulmonary bypass (CPB). Nine patients had DeBakey type I and 5 had DeBakey type II aortic dissection. CPB was initiated with aortic cannulation at the dissected site in all but 4 of the patients with DeBakey type II aortic dissection. There were 4 female and 6 male patients aged $59 \pm 11$ years (range, 41-77 years). Two patients had sealed rupture of the ascending aorta, and one of them had a pericardial effusion.

After median sternotomy and longitudinal pericadiotomy, a less dissected or nondissected cannulation site is cautiously sought and determined by means of palpation and transesophageal echocardiography. By using normal double-purse-string sutures, the aortic 